\title{
LAPAROSCOPIC APPENDECTOMY VERSUS OPEN APPENDECTOMY IN CHILDREN: EVOLUTION OVER TIME
}

\author{
LAURA DUMITRICÄ ${ }^{1}$, LUMINIŢA DOBROTÄ ${ }^{2}$, BOGDAN NEAMȚU \\ ${ }^{1,3}$ Pediatric Clinic Hospital Sibiu, ${ }^{2}$ "Lucian Blaga” University of Sibiu, \\ ${ }^{3}$ Pediatric Clinic-Research and Telemedicine Center in Neurological Diseases in Children, Sibiu
}

\begin{abstract}
Keywords: appendicitis, Abstract: Laparoscopic appendicitis surgery is accepted in more and more centers around the world. laparoscopic appendectomy,

open appendectomy, Studies and meta-analyses of studies have shown that laparoscopic appendicitis is a feasible and safe laparoscopy, comparative evaluation procedure with numerous clinical benefits, such as shorter postoperative ileus, lower incidence of wound infection, lower postoperative pain and duration, recurrence faster to activities. Because laparoscopic appendectomy has been associated with a reduced risk of surgical complications, it may provide a better alternative versus open surgery. A review of data relevant to the evaluation of laparoscopic appendectomy versus open appendectomy as reflected in the literature of the last 2 decades would be relevant for the growing progressive interest of laparoscopic surgery for acute appendicitis and for its comparative evaluation with classical open appendicitis intervention.
\end{abstract}

\section{INTRODUCTION}

Laparoscopic appendicitis (LAP) surgery is accepted in more and more centres around the world. Studies and metaanalyses of studies have shown that LA is a feasible and safe procedure with numerous clinical benefits, such as shorter postoperative ileus, lower incidence of wound infection, lower postoperative pain and duration, recurrence faster to activities.

A review of data relevant to the evaluation of laparoscopic appendectomy (LA) vs. Open appendectomy (OA) as reflected in the literature of the last 2 decades would be relevant for the increasing progressive interest of laparoscopic surgery for acute appendicitis and for its comparative evaluation with classical open OA intervention.

\section{AIM}

The main aim consists in a brief review of the relevant data for the stated topic as they are reflected in the literature of the last 2 decades as well as highlighting the progress of LA and how this surgical technique has been evaluated compared to OA, evolving in the last 2 decades.

\section{MATERIALS AND METHODS}

Consulting the specialized medical literature regarding the stated topic using as search keywords: laparoscopic appendectomy, open appendectomy, comparative evaluation.

Selecting the significant studies for the last 20 years, a selection based on the relevance of the study for the comparative evaluation of LA vs OA and the evolution over time of this concept of the comparative evaluation of the two surgical techniques.

\section{RESULTS AND DISCUSSIONS}

In 2004, the question of the opportunity of laparoscopic appendectomy was still raised when it was discussed in comparison with the classic intervention, respectively with the open appendectomy.

In this context, Hitoshi Ykeda concluded that laparoscopic appendectomy should remain an option in both children with uncomplicated appendicitis and those with complicated appendicitis. The author pleads for this option which, although it was more expensive than the classic intervention and requires a longer operating time, was distinguished from it by the shorter duration of hospitalization (for those with uncomplicated appendicitis).(1)

In both types of appendicitis, uncomplicated and complicated too, there were no significant differences between laparoscopic and open appendectomies in terms of complications or their incidence.

Finally, the author concludes that LA may remain an option provided that the advantages and disadvantages of the procedure are considered.(1)

In the same context, 7 years later (2011), Ching-Chung Tsai et al. conducted a study in which they aim to answer the question of whether laparoscopic appendectomy is an alternative therapeutic tool to classical open appendectomy for all types of appendicitis, respectively simple, perforated and with abscess.(2)

The authors conclude that laparoscopic appendectomy can be considered a safe alternative for both perforated appendicitis and abscessed appendicitis. The laparoscopic intervention results in statistically significant postoperative with fewer minor complications for perforated appendicitis and with fewer major complications for abscessed appendicitis compared to open (classic) appendectomy, $(9 / 32$ compared to $0 / 20, p=0.009)$ and respectively $(9 / 26$ compared to $1 / 24, \mathrm{p}=0.011) .(2)$

In the same year, Steven L. Lee published a retrospective cohort study that included 7.650 children, 3.551 with LA and 4.099 with OA.(3) Assuming that the results of LA will be similar to OA in children of all ages, however, finds that LA is the preferred operation for children with appendicitis, because LA compared to OA was associated with a low risk of wound infection, abscess drainage and length of hospital stay.(3)

In the study signed by Hossein Masoomi et al. in 2012 (USA) the authors aim to explore the benefits of laparoscopic appendectomy on a case series of 212.958 children with

${ }^{2}$ Corresponding author: Luminiţa Dobrotă, Str. Lucian Blaga, Nr. 2A, Sibiu, Romania, E-mail: luminitadobrota@yahoo.com, Phone: +40722501145 Article received on 01.09.2020 and accepted for publication on 26.02.2021 


\section{CLINICAL ASPECTS}

emergency operated appendicitis over a period of 2 years, according to 2006-2008 Data from The Nationwide Inpatient Sample (NIS).(4) In cases of non-perforated appendicitis, LA was associated with a percentage of comparable overall complications (LA, $2,56 \%$ vs OA, $2.66 \%$; p 0,26), shorter length of hospital stay (LA, 1,6 days vs OA, 2,0 days; $\mathrm{p}<0,01$ ), comparable mortality (LA, $0,01 \%$ vs OA, $0,02 \%$; p 0,25) and higher hospital fees (LA, $20,328$ USD vs. OA, 16,830 USD; $p<0,01)$. In cases of perforated appendicitis, LA was associated with a lower percentage of general complications (LA, $16,03 \%$ vs OA, 18,07\%; $\mathrm{p}<0,01$ ), shorter length of hospital stay (LA, 5,1 days vs OA, 5,8 days; $\mathrm{p}<0,01$ ), lower mortality (LA, $0,0 \%$ vs OA, $0,06 \%$; $\mathrm{p}<$ 0.01 ) and similar hospital expenses (LA, 33,361 USD compared to OA, 33, 662 USD, $\mathrm{p}=0,71)$.(4)

The authors conclude that LA (performed in $56,9 \%$ of cases) is:

- safe in the case of children with acute perforated or nonperforated appendicitis;

associated with a shorter hospital stay than OA;

associated with lower complications and mortality than OA in both non-perforated and perforated appendages.(4)

It is mentioned, however, that in cases of non-perforated appendicitis, the benefits are modest and associated with higher hospitalization costs.(4)

Assuming that LA or open OA are associated with hospital procedural preferences, Jun Tashiro et al. in 2016, consulted the database for children hospitalized in the USA during 1997-2009 for simple appendicitis and complicated.(5) The authors evaluated LA vs. OA comparatively according to a series of parameters and complications (symptoms, duration of hospitalization, infusion treatment, surgical wound infections, perforations/lacerations, total hospitalization costs) in two different hypostases of simple and complicated appendicitis.(5)

Selective analysis of simple appendicitis (91.118 cases with LA vs 97.496 with OA), LA vs OA generated the following data: an increased percentage of infusions $(1,7 \%)$,

fewer wound infections $(0,6 \%)$,

a lower percentage of perforations/lacerations $(0,3 \%)$,

a shorter length of hospital stay (1,7 versus 2,1 days) but higher total hospitalization costs (19.501 USD vs. 13.089 USD).

For complicated appendicitis (28.793 cases with LA vs. 30.782 with OA), LA vs. OA presented:

higher percentage of nausea/vomiting $(1,9 \%)$,

lower percentage of surgical wound infections $(0,5 \%)$ and cases that required infusions $(0,6 \%)$.

shorter length of hospital stay (5,1 vs. 5,9 days) but higher total costs ( 32.251 vs. 28.209 USD).

The discussions, results and conclusions outline the following aspects: complications and the use of appendicitis resources are associated with surgical technique and hospital procedure preferences.

Hospitals that prefer laparoscopic interventions had:

higher percentages of complications with the OA technique for complicated appendicitis, and

higher expenses, regardless of the technique of appendectomy or the type of appendicitis.

Comparing "Laparoscopic vs. Open Appendectomy in children with complicated appendicitis, 2017" authors of the mentioned study, Mohammad G. Kirallah et al., concludes that LA vs. OP was an appropriate, efficient and safe procedure in complicated cases, in addition to being associated with an operating time and a lower percentage of complications.(6)

Also, in 2017, Liping Dai et al published in the United European Gastroenterology Journal a meta-analysis of controlled and randomized studies.(7) The authors aim to evaluate LA vs OA in adults and children in terms of the following criteria: type of appendicitis, postoperative complications, percentage of operative re-interventions, operative time, duration of postoperative hospitalization, return to normal activity. The analysis of the subgroups of children did not show significant differences between the two techniques in terms of wound infection, postoperative complications, duration of postoperative hospitalization and return to normal activity.(7)

At the end of the study, it is concluded that highperformance LA in adults can be recommended as an effective and safe procedure for acute appendicitis, but for children additional high-quality randomized studies comparing the two techniques would be needed.(7)

Roshan Ali et al., in 2017, addresses the comparative assessment of LA vs OA in a controlled and randomized study in developing countries.(8) The study proposes this evaluation according to the following parameters: length of hospital stay, operative time and postoperative wound infection. The results did show that the operative time was $56 \pm 24$ minutes in the LA group and $39 \pm 8$ minutes in the OA group ( $\mathrm{p}<0,0001$ in favor of OA); the mean length of hospital stay was $34 \pm 13$ hours for the LA group and $40 \pm 11$ hours for the OA group ( $p=0,01$ in favor of LA). The results did not show a significant association of wound infection between the two groups $(p=0,31)$. In conclusion, it is specified that no statistically significant differences were found between the 2 groups in terms of length of hospital stay and postoperative wound infection. However, the authors mention, the laparoscopic procedure is more technically difficult.(8)

Ping Li et al., in 2017, publishes an article presenting a retrospective review of patients operated on by the two techniques for abscessed appendicitis between 2005 and 2016, therewith proposing a comparative assessment of recovery and postoperative complications in LA versus OA.(9) Patients with LA received a rapid postoperative recovery of gastrointestinal function, such as the first bowel movement $(\mathrm{RR}=0,52[95 \% \mathrm{CI}$ $0,44-0,69] \mathrm{p}<0,001$, respectively $\mathrm{RR}=0,53$ [95\% CI, 0,41-0,76] $\mathrm{p} 0,001)$ compared to patients with OA. In addition, the number of leukocytes $(\mathrm{RR}=0,56[95 \% \mathrm{CI}, 0,46-0,73] \mathrm{p}<0,001)$ and $\mathrm{C}$ reactive protein, $\mathrm{CRP}(\mathrm{RR}=0,58[95 \% \mathrm{CI} 0,86] \mathrm{p} 0,11)$ as postoperative inflammatory tests recorded lower values in patients with LA vs. patients with OA. A general percentage of postoperative complications, including surgical wound infection $(\mathrm{OR}=0,38 ; 95 \% \mathrm{CI} ; 0,18-0,81 ; \mathrm{p} 0,008)$ and incision dehiscence $(\mathrm{OR}=0,06 ; 95 \% \mathrm{CI} ; \mathrm{p}<0.001)$ was observed in patients with LA compared to OA. LA has been feasible and effective for abscessed appendicitis and associated with beneficial clinical effects, such as recovery of postoperative gastrointestinal function and reduced postoperative complications. LA should be seriously considered to be the first line of choice.(9)

Kengo Inagaki et al., in 2019, publishes in the Journal of Surgical Research a study that aims to evaluate the associated factors, complications and re-admissions related to the 2 types of LA and OA interventions, stating that the particularities of the patient with $\mathrm{OA}$ are weak characterized.(10)

Factors associated with $\mathrm{OA}$ were the percentage of readmission after 30 days and the length of hospital stay.

Of the 46.147 children operated for appendicitis, $85,2 \%$ benefited from LA. Laparoscopic appendectomy was associated with a shorter length of hospital stay (incidence ratio: 0,77 [95\% CI: $0,69-0,87]$ ), fewer re-admissions with wound infection, without being motivated by readmission after 30 days or readmission for intra-abdominal abscess.

Open appendectomy has been associated with smaller hospitals (OR: 3,01 [95\% CI: 1,81-5,01]), rural hospitals (OR: 2,36 [95\% CI: 1,63-3,40]), public insurance (OR: 1,19 [95\% CI: 1,03-1,36]), lower income neighbourhood residence (OR: 1,40 [95\% CI: $1,06-1,86])$, younger than 5 years old of age (OR: 5,00 [95\% CI: 3,64-6,86], complicated - abscessed appendicitis (OR: 


\section{CLINICAL ASPECTS}

1,91 [IC 95\%: 1,58-2,31]). The conclusions of the study show on the one hand how clinical factors and the rural/didactic status of treatment hospitals play a role in choosing the surgical approach, and in the other hand how awareness of patient and hospitalrelated factors associated with OA may influence resource allocation or improve access to in-care.

Zhi Xuan Low et al., in 2019, start from the premise that LA is preferred vs. OA in patients with uncomplicated appendicitis. However, for patients with complicated appendicitis (suppurative, gangrenous or perforated or with periappendicular abscess formation), the decision to perform OA or LA remains unclear.(11) Following the PRISMA guidelines, the search in databases from 1997 to 2017 (Cochrane, Medline, PubMed, Scopus, Ovidiu, Embase and Web of Knowledge) and the analysis of the study subgroups of randomized control, was performed using RevMan 5.3. The assessment of methodological and statistical heterogeneity was performed, and 7 randomized control studies (296 LA versus 373 OA) and 33 control case studies (3.106 LA versus $4.149 \mathrm{OA}$ ) were analysed. LA vs OA has a shorter hospital stay (weighted mean difference - WMD $=-0,96$ [95\% CI 1,47-0,45]) and a lower rate of operative wound infection (OR 0,37 [95\%CI 0,25-0,54]), although the percentages of intraabdominal abscess formation were similar (OR 1,01 [95\% CI $0,71-1,43])$. LA had lower readmission rates, lower incidence of postoperative ileus or intestinal obstruction, lower incidence of need for reoperation, and a shorter time to resume oral intake. The operative time for OA was shorter than LA (WMD $=12,44[95 \%$ CI 2,00-22,87]). The conclusion of the study LA should be the procedure of choice for pediatric patients with complicated appendicitis because the percentages of postoperative intraabdominal abscess are similar for both techniques.(11)

Ritvik Resutra and Rajive Gupta, in 2020, publish in the International Journal of Minimal Access Surgery a study evaluating the technical feasibility and safety of LA in acute appendicitis versus the results obtained with OA.(12) A total of 400 pediatric patients with acute appendicitis, 200 by LA and 200 by $\mathrm{OA}$ were operated on by a single surgeon at various private hospitals in Jammu and Kashmir, India, between June 2017 and May 2020. The following were evaluated: operating time, duration of hospitalization, postoperative pain, percentage of complications, time required to resume activity and patient satisfaction correlated with the cosmetics of the intervention. Better results with LA (vs. OA) were: significantly lower postoperative pain, faster recovery, resumption of activities earlier, reduction of postoperative complications and better cosmetic patient satisfaction. The conclusion of the study was that the LA is a safe and feasible technique for experts, results comparable to OA, without an increase in complications being the procedure of choice for the treatment of acute appendicitis.

\section{CONCLUSIONS}

Laparoscopic appendicitis surgery is accepted in more and more centres around the world. Studies and meta-analyses of studies have shown that LA is a feasible and safe procedure with many clinical benefits, such as: shorter postoperative ileus, lower incidence of wound infection, lower postoperative pain, and faster return to normal work activities.

Because LA has been associated with a reduced risk of surgical complications, it may provide a better alternative vs. OP is currently a first choice option.

In the age group specific to younger children, there are not yet enough studies to evaluate LA vs OP. In this regard, additional high-quality randomized studies are needed to compare the two techniques in children.

Acknowlegdement:

Part of the analysis has been conducted in the Pediatric Clinic Hospital Sibiu, within Research and Telemedicine Center in Neurological Diseases in Children - CEFORATEN project (ID928 SMIS-CSNR 13605) financed by ANCSI with the grant number 432 / 21. 12. 2012 thru the Sectoral Operational Programme ,Increase of Economic Competitiveness".

\section{REFERENCES}

1. Ikeda H, Ishimaru Y, Takayasu H, Okamura K, Kisaki Y, Kosighaya JF. Laparoscopic versus open appendectomy in children with uncomplicated and complicated appendicitis; Journal of Pediatric Surgery. 2004;39(11):1680-1685, https://doi.org/10.1016/j.jpedsurg.2004.07.018.

2. Tsai CC, Lee SY, Huang FC. Laparoscopic versus Open Appendectomy in the Management of all stages of acute appendicitis in children: a retrospective study; Pediatrics and Neonatology. 2012;53:289e294 http://dx.doi.org/10.1016/j.pedneo.2012.07.002.

3. Lee SL, Yaghoubian A, Kaji A. Laparoscopic vs Open Appendectomy in Children, Outcomes Comparison Based on Age, Sex, and Perforation Status; Arch Surg. 2011;146(10):1118-1121. Published online June 20, 2011. doi:10.1001/archsurg.2011.144.

4. Masoomi H, Mills S, Dolich MO, Ketana N, Carmichael JC, Nguyen NT, Stamos MJ. Comparison of Outcomes of Laparoscopic Versus Open Appendectomy in Children: Data from The Nationwide Inpatient Sample (NIS), 2006-2008; World Journal of Surgery March 2012;36(3):573-578.

5. Tashiro J, Einstein SA, Perez EA, Bronson SN, Lasko DS, Sola JE. Hospital preference of laparoscopic versus open appendectomy: Effects on outcomes in simple and complicated appendicitis; jpedsurg. 2016.02.028, https://doi.org/10.1016/j.

6. Kirallah MG, Eldesouki NI, Elzanaty AA, Ismail KA. Laparoscopic versus open appendectomy in children with complicated appendicitis; Annals of Pediatric Surgery. 2017;13(1):17-20. January 2017, DOI: 10.1097/01.XPS.0000496987.42542.dd.

7. Dai L, Shuai J. Laparoscopic versus open appendectomy in adults and children: A meta-analysis of randomized controlled trials; Gastroenterology Journal 2017;5(4):542553, DOI: $10.1177 / 2050640616661931$.

8. Roshan A, et al. Laparoscopic versus open appendectomy in children: a randomized controlled trial from a developing country; J Pediatr Surg. 2018 Feb;53(2):247-249. doi:10.1016/j.jpedsurg.2017.11.022. Epub 2017 Nov 14.

9. Li P, Han Y, Yang Y, Guo H, Hao F, Tang Y, Guo C. Retrospective review of laparoscopic versus open surgery in the treatment of appendiceal abscess in pediatric patients; Medicine (Baltimore). 2017 Jul;96(30):e7514. doi: 10.1097/MD.0000000000007514.

10. Inagaki K, Blackshear C, Morris MW, Hobbs CV. Pediatric Appendicitis-Factors Associated With Surgical Approach, Complications, and Readmission; Journal of Surgical Research; October 16, 2019 , https://doi.org/10.1016/j.jss.2019.09.031

11. Low ZX, Bonney GK, So JBY, Loh DL, Ng JJ. Laparoscopic versus open appendectomy in pediatric patients with complicated appendicitis: a meta-analysis; Surg Endosc. 2019 Dec;33(12):4066-4077. doi: 10.1007/s00464-01906709-x. Epub 2019 Feb 25.

12. Resutra R, Gupta R. Comparative Study of Laparoscopic Appendectomy versus Open Appendectomy for the Treatment of Acute Appendicitis. International Journal of Minimal Access Surgery, 2020, Volume 1| Article 1005. 